\title{
Effect of Thickness Stretching on the Natural Frequencies of Laminate- Faced Sandwich Plates Using a New Layerwise Model
}

\author{
Belarbi MO1,*, Tati A', Khechai $\mathrm{A}^{2}$
}

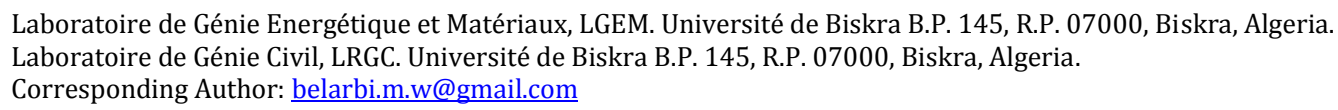

Received: 30-04-2019

Accepted: 17-07-2019

\begin{abstract}
The current work investigates the effect of thickness stretching on the natural frequencies of laminate-faced sandwich plates using new layerwise finite element model. The proposed model assumes higher-order displacement field for the core and first-order displacement field for the face sheets. Thanks for enforcing the continuity of the interlaminar displacement, the number of variables is independent of the number of layers. The consistent mass matrix and the element stiffness matrix are derived using the Hamilton's principle. The performance and reliability of the proposed formulation are demonstrated by comparing the author's results with those obtained using the three-dimensional elasticity theory, analytical solutions and other advanced finite element models.
\end{abstract}

Key words: Layerwise, Finite element, Sandwich plates, Free vibration.

\section{Introduction}

Composite sandwich structurers provide high performance and reliability due to their low weight, high stiffness and high strength properties. As a result, composite structures, such as sandwichs plates, will continue to be widely used for many years in the engineering fields such as civil, naval, aerospace and construction industries. Despite the many advantages of sandwich structures, their behavior becomes very complex due to the large variation of rigidity and material properties between the core and the face sheets.

Different plate theories have been proposed to study the behavior of sandwich structures. These plate theories may be grouped as equivalent single layer (ESL) approach (where all the layers are referred to the same variables) and layerwise (LW) approach. The ESL approach can be divided into three major theories, namely: (1) the classical laminated plate theory (CLPT); (2) the first order shear deformation theory (FSDT); and (3) the higher order shear deformation theories (HSDT).

However, ESL approach fail to capture precise the local behavior of sandwich structures. This drawback in ESL was circumvented by the layerwise theories in which the displacements are assumed at the mid surface of each laminate and maintaining the continuity of the displacements at the layer interface (Pandey \& Pradyumna, 2015).

In the finite elements (FE) development, many researchers have adopted the LW approach for the sake of a good description of sandwich structures (Belarbi \& Tati, 2015; Belarbi et al., 2016; Belarbi \& Tati, 2016). On this topic, we can distinguish the work of Nabarrete et al. (2003), where a 3D layerwise FE model is developed for free vibration analysis of sandwich plates. They used the FSDT to model the face sheets and the HSDT was adopted to model the core. Desai (Desai et al., 2003) developed an eighteen-node layerwise mixed brick element with 108 degrees-of-freedom (DOFs) for the free vibration analysis of multi-layered thick composite plates. Later, an eight nodes quadrilateral element having 136 DOFs was developed by Araújo et al. (2010) for the analysis of sandwich laminated plates with a viscoelastic core and laminated anisotropic face layers. The construction of this element is based on layerwise approach where 
the HSDT is used to model the core layer and the face sheets are modeled according to a FSDT. Elmalich \& Rabinovitch (2012) have undertaken an analysis on the dynamics of sandwich plates, using a C0 four-node rectangular element. The formulation of this element is based on the use of a new layerwise model, where the FSDT is used for the face sheets and the HSDT is used for the core. More recently, Pandey \& Pradyumna (2015) presented a new higher-order layerwise plate formulation for static and free vibration analyses of laminated composite plates. A high order displacement field is used for the middle layer and a first-order displacement field for top and bottom layers. The authors used an eight-noded isoparametric element containing 104 DOFs to model the plate.

The goal of this work is to propose a new 2D layerwise FE formulation for free vibration analyses of multi-layered sandwich plates. Unlike layerwise models, the number of variables in the present model is independent of the number of layers. The results obtained from this investigation will be useful for a more understanding of the free vibration behavior of sandwich laminates plates.

\section{Mathematical Formulation}

Sandwich plate is a structure composed of three principal layers as shown in Fig.1, two face sheets (top-bottom) of thicknesses $\left(h_{t}\right),\left(h_{b}\right)$ respectively, and a central layer named core of thickness $\left(h_{c}\right)$ which is thicker than the previous ones. Total thickness $(h)$ of the plate is the sum of these thicknesses. The plane $(x, y)$ coordinate system coincides with mi-plane plate.

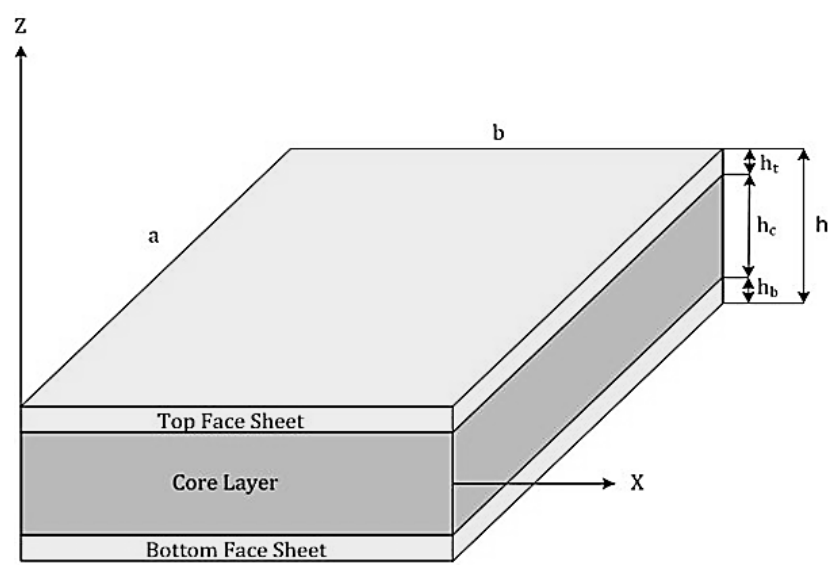

Fig 1. Geometry and notations of a sandwich plate.

In the present model, the HSDT is adopted for the core layer. Hence, the displacement field is written as a third-order Taylor series expansion of the in-plane displacements in the thickness coordinate, and as a constant one for the transverse displacement:

$$
\begin{aligned}
& u_{c}=u_{0}+z \psi_{x}^{c}+z^{2} \eta_{x}^{c}+z^{3} \zeta_{x}^{c} \\
& v_{c}=v_{0}+z \psi_{y}^{c}+z^{2} \eta_{y}^{c}+z^{3} \zeta_{y}^{c} \\
& w_{c}=w_{0}
\end{aligned}
$$

where $u_{0}, v_{0}$ and $w_{0}$ are in-plane and transverse displacement components at the mid-plane of the sandwich plate, respectively. $\psi_{x}^{c}$ and $\psi_{y}^{c}$ represent normal rotations about the $x$ and $y$ axis respectively. The parameters $\eta_{x}^{c}, \eta_{y}^{c}, \zeta_{x}^{c}$ and $\zeta_{y}^{c}$ are higher-order terms the Taylor's series expansion.

\subsection{Strain-displacement relations}

The kinematic relations for the core are given by: 


$$
\begin{aligned}
& \varepsilon_{x x}^{c}=\frac{\partial u_{0}}{\partial x}+z \frac{\partial \psi_{x}^{c}}{\partial x}+z^{2} \frac{\partial \eta_{x}^{c}}{\partial x}+z^{3} \frac{\partial \zeta_{x}^{c}}{\partial x} \\
& \varepsilon_{y y}^{c}=\frac{\partial v_{0}}{\partial y}+z \frac{\partial \psi_{y}^{c}}{\partial y}+z^{2} \frac{\partial \eta_{y}^{c}}{\partial y}+z^{3} \frac{\partial \zeta_{y}^{c}}{\partial y} \\
& \gamma_{x y}^{c}=\left(\frac{\partial u_{0}}{\partial y}+\frac{\partial v_{0}}{\partial x}\right)+z\left(\frac{\partial \psi_{x}^{c}}{\partial y}+\frac{\partial \psi_{y}^{c}}{\partial x}\right)+z^{2}\left(\frac{\partial \eta_{x}^{c}}{\partial y}+\frac{\partial \eta_{y}^{c}}{\partial x}\right)+z^{3}\left(\frac{\partial \zeta_{x}^{c}}{\partial y}+\frac{\partial \zeta_{y}^{c}}{\partial x}\right) \\
& \gamma_{y z}^{c}=\psi_{y}^{c}+\frac{\partial w_{0}}{\partial y}+z 2 \eta_{y}^{c}+z^{2} 3 \zeta_{y}^{c} \\
& \gamma_{x z}^{c}=\psi_{x}^{c}+\frac{\partial w_{0}}{\partial x}+z 2 \eta_{x}^{c}+z^{2} 3 \zeta_{x}^{c}
\end{aligned}
$$

\subsection{Displacement field of the face sheets}

The face sheets are modeled using the FSDT. The compatibility conditions as well as the interlaminar displacement continuity (face sheets/core), leads to the following improved displacement fields:

Top face sheet

$$
\begin{aligned}
& u_{t}=u_{0}+\left(\frac{h_{c}}{2}\right) \psi_{x}^{c}+\left(\frac{h_{c}^{2}}{4}\right) \eta_{x}^{c}+\left(\frac{h_{c}^{3}}{8}\right) \zeta_{x}^{c}+\left(z-\frac{h_{c}}{2}\right) \psi_{x}^{t} \\
& v_{t}=v_{0}+\left(\frac{h_{c}}{2}\right) \psi_{y}^{c}+\left(\frac{h_{c}^{2}}{4}\right) \eta_{y}^{c}+\left(\frac{h_{c}^{3}}{8}\right) \zeta_{y}^{c}+\left(z-\frac{h_{c}}{2}\right) \psi_{y}^{t} \\
& w_{t}=w_{0}
\end{aligned}
$$

Bottom face-sheet

$$
\begin{aligned}
& u_{b}=u_{0}-\left(\frac{h_{c}}{2}\right) \psi_{x}^{c}+\left(\frac{h_{c}^{2}}{4}\right) \eta_{x}^{c}-\left(\frac{h_{c}^{3}}{8}\right) \zeta_{x}^{c}+\left(z+\frac{h_{c}}{2}\right) \psi_{x}^{b} \\
& v_{b}=v_{0}-\left(\frac{h_{c}}{2}\right) \psi_{y}^{c}+\left(\frac{h_{c}^{2}}{4}\right) \eta_{y}^{c}-\left(\frac{h_{c}^{3}}{8}\right) \zeta_{y}^{c}+\left(z+\frac{h_{c}}{2}\right) \psi_{y}^{b} \\
& w_{b}=w_{0}
\end{aligned}
$$

\subsection{Strain -displacement relations}

The kinematic relations for the top face sheet can be written as follows:

$$
\begin{aligned}
& \varepsilon_{x x}^{t}= \frac{\partial u_{t}}{\partial x}=\frac{\partial u_{0}}{\partial x}+\left(\frac{h_{c}}{2}\right) \frac{\partial \psi_{x}^{c}}{\partial x}+\left(\frac{h_{c}^{2}}{4}\right) \frac{\partial \eta_{x}^{c}}{\partial x}+\left(\frac{h_{c}^{3}}{8}\right) \frac{\partial \zeta_{x}^{c}}{\partial x}+\left(z-\frac{h_{c}}{2}\right) \frac{\partial \psi_{x}^{t}}{\partial x} \\
& \varepsilon_{y y}^{t}=\frac{\partial v_{t}}{\partial y}=\frac{\partial v_{0}}{\partial y}+\left(\frac{h_{c}}{2}\right) \frac{\partial \psi_{y}^{c}}{\partial y}+\left(\frac{h_{c}^{2}}{4}\right) \frac{\partial \eta_{y}^{c}}{\partial y}+\left(\frac{h_{c}^{3}}{8}\right) \frac{\partial \zeta_{y}^{c}}{\partial y}+\left(z-\frac{h_{c}}{2}\right) \frac{\partial \psi_{y}^{t}}{\partial y} \\
& \gamma_{x y}^{t}=\frac{\partial u_{t}}{\partial y}+\frac{\partial v_{t}}{\partial x}=\left(\frac{\partial u_{0}}{\partial y}+\frac{\partial v_{0}}{\partial x}\right)+\frac{h_{c}}{2}\left(\frac{\partial \psi_{x}^{c}}{\partial y}+\frac{\partial \psi_{y}^{c}}{\partial x}\right)+\frac{h_{c}^{2}}{4}\left(\frac{\partial \eta_{x}^{c}}{\partial y}+\frac{\partial \eta_{y}^{c}}{\partial x}\right) \\
&+\frac{h_{c}^{3}}{8}\left(\frac{\partial \zeta_{x}^{c}}{\partial y}+\frac{\partial \zeta_{y}^{c}}{\partial x}\right)+\left(z-\frac{h_{c}}{2}\right)\left(\frac{\partial \psi_{x}^{t}}{\partial y}+\frac{\partial \psi_{y}^{t}}{\partial x}\right) \\
& \gamma_{y z}^{t}=\frac{\partial w_{0}}{\partial y}+\psi_{y}^{t}, \quad \gamma_{x z}^{t}=\frac{\partial w_{0}}{\partial x}+\psi_{x}^{t}
\end{aligned}
$$


The same steps are followed to elaborate the strain-displacement relationships of the bottom face sheet.

\subsection{Constitutive Relationships}

In this work, the two face sheets (top and bottom) are considered as laminated composite. Hence, the stress-strain relations for $k^{\text {th }}$ layer in the global coordinate system are expressed as:

$$
\left\{\begin{array}{l}
\sigma_{x x}^{f} \\
\sigma_{y y}^{f} \\
\tau_{y z}^{f} \\
\tau_{x z}^{f} \\
\sigma_{x y}^{f}
\end{array}\right\}^{(k)}=\left[\begin{array}{ccccc}
\overline{Q_{11}} & \overline{Q_{12}} & 0 & 0 & \overline{Q_{16}} \\
\overline{Q_{21}} & \overline{Q_{22}} & 0 & 0 & \overline{Q_{26}} \\
0 & 0 & \overline{Q_{44}} & \overline{Q_{45}} & 0 \\
\overline{0} & 0 & \overline{Q_{54}} & \overline{Q_{55}} & 0 \\
\overline{Q_{61}} & 0 & 0 & \overline{Q_{66}}
\end{array}\right]^{(k)}\left\{\begin{array}{c}
\varepsilon_{x x}^{f} \\
\varepsilon_{y y}^{f} \\
\gamma_{y z}^{f} \\
\gamma_{x z}^{f} \\
\gamma_{x y}^{f}
\end{array}\right\} \quad f=\text { top, bottom }
$$

The core is considered as an orthotropic composite material, and the stress-strain relationships can be defined as follows:

$$
\left\{\begin{array}{c}
\sigma_{x x} \\
\sigma_{y y} \\
\tau_{y z} \\
\tau_{x z} \\
\sigma_{x y}
\end{array}\right\}=\left[\begin{array}{ccccc}
\overline{Q_{11}} & \overline{Q_{12}} & 0 & 0 & \overline{Q_{16}} \\
\overline{Q_{21}} & \overline{Q_{22}} & 0 & 0 & \overline{Q_{26}} \\
0 & 0 & \overline{Q_{44}} & \overline{Q_{45}} & 0 \\
\overline{0} & 0 & \overline{Q_{54}} & \overline{Q_{55}} & 0 \\
\overline{Q_{61}} & \overline{Q_{62}} & 0 & 0 & \overline{Q_{66}}
\end{array}\right]\left\{\begin{array}{c}
\varepsilon_{x x} \\
\varepsilon_{y y} \\
\gamma_{y z} \\
\gamma_{x z} \\
\gamma_{x y}
\end{array}\right\}
$$

The efforts resultants of the core are obtained by integration of the stresses through the thickness direction of laminated plate. Hence, the constitutive equations can be written in the following contracted form:

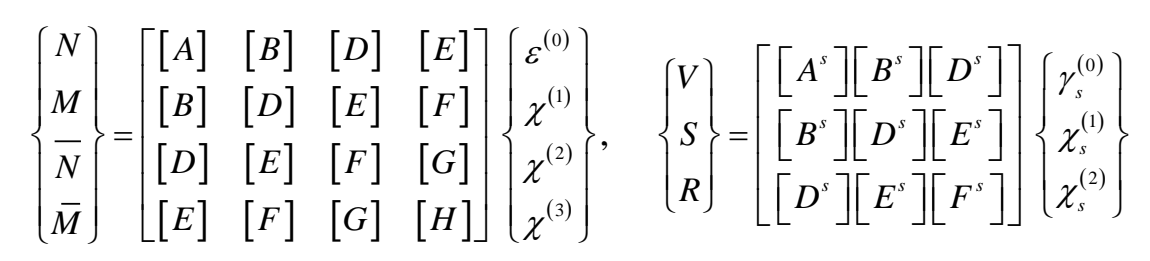

where the components of the reduced stiffness matrices of the core are defined by:

$$
\begin{array}{ll}
\left(A_{i j}, B_{i j}, D_{i j}, E_{i j}, F_{i j}, G_{i j}, H_{i j}\right)=\int_{\frac{-h_{c}}{2}}^{\frac{h_{c}}{2}} Q_{i j}\left(1, z, z^{2}, z^{3}, z^{4}, z^{5}, z^{6}\right) d z & (i, j=1,2,6) \\
\left(A_{i j}^{s}, B_{i j}^{s}, D_{i j}^{s}, E_{i j}^{s}, F_{i j}^{s}\right)=\int_{\frac{-\frac{h_{c}}{2}}{2}}^{\frac{h_{c}}{2}} Q_{i j}\left(1, z, z^{2}, z^{3}, z^{4}\right) d z & (i, j=4,5)
\end{array}
$$

According to the theory FSDT, the constitutive equations for the face sheets are:

$$
\left\{\begin{array}{c}
N^{f} \\
M^{f} \\
T^{f}
\end{array}\right\}=\left[\begin{array}{ccc}
A^{f} & B^{f} & 0 \\
B^{f} & D^{f} & 0 \\
0 & 0 & A_{c}^{f}
\end{array}\right]\left\{\begin{array}{l}
\varepsilon_{m}^{f} \\
\varepsilon_{f}^{f} \\
\gamma_{c}^{f}
\end{array}\right\}
$$




\section{Finite Element Formulation}

In the present study, a C0 four-node isoparametric element, named QSFT52 (Quadrilateral Sandwich First Third with 52-DOF), with thirteen DOF per node has been developed. Each node contains: two rotational DOF for each face sheet, six rotational DOF for the core, while the three translations DOF are common for sandwich layers (figure 2).

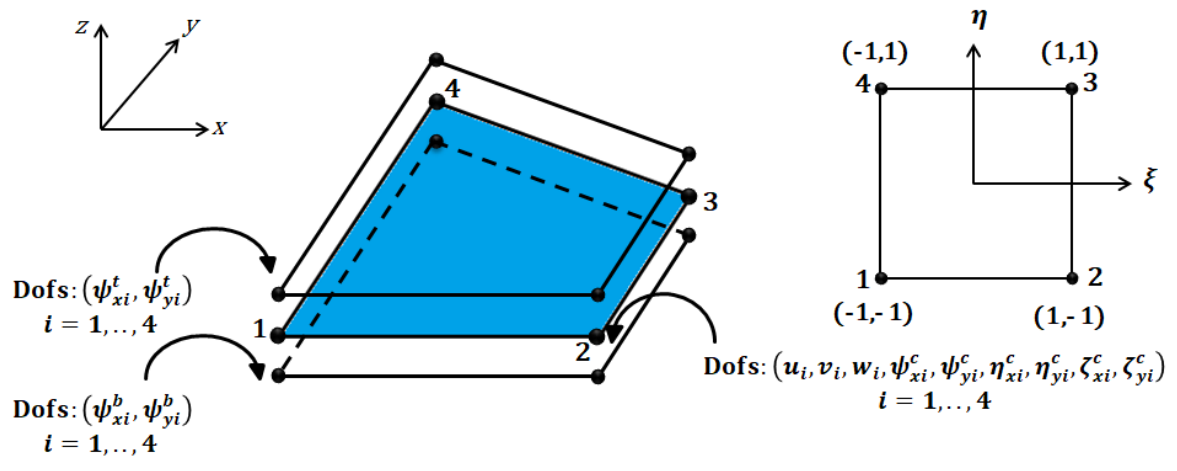

Fig 2. Geometry and corresponding degrees of freedom of the QSFT52 element.

The displacements vectors at any point of coordinates $(x, y)$ of the plate are given by:

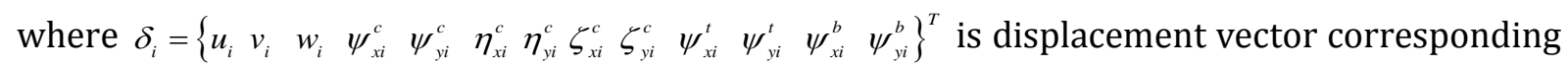
to node $i(i=1,2,3,4)$.

The generalized strain vector for three layers can be expressed in terms of nodal displacements vector as follows:

$$
\left\{\varepsilon^{(k)}\right\}=\left[B_{i}^{(k)}\right]\left\{\delta_{i}\right\}
$$

\subsection{Governing Differential Equation}

In this work, Hamilton's principle is applied in order to formulate governing free vibration problem, which is given as:

$$
\delta \Pi=\delta \int_{t_{1}}^{t_{2}}(T-U) d t=0
$$

where $t$ is the time, $T$ is the kinetic energy of the system and $U$ is the potential energy of the system.

Using the standard finite element procedure, the governing differential equations of motion can be rewritten as:

$$
\left[M_{T}\right]\{\ddot{\delta}\}+\left[K_{T}\right]\{\delta\}=0
$$

where $\left[M_{T}\right]$ and $\left[K_{T}\right]$ denote the element mass matrix and the element stiffness matrix, respectively, for the three layers sandwich plate.

$$
[K]=\sum_{e} \int_{-1}^{1} \int_{-1}^{1}\left(\left[B^{t}\right]^{T}\left[D^{t}\right]\left[B^{t}\right]+\left[B^{c}\right]^{T}\left[D^{c}\right]\left[B^{c}\right]+\left[B^{b}\right]^{T}\left[D^{b}\right]\left[B^{b}\right]\right) \operatorname{det}(J) d \xi d \eta
$$

and the element mass matrix can be written as:

$$
[M]=\sum_{e} \int_{-1}^{1} \int_{-1}^{1}\left([N]^{T}\left[m^{(t)}\right][N]+[N]^{T}\left[m^{(c)}\right][N]+[N]^{T}\left[m^{(b)}\right][N]\right) \operatorname{det}(J) d \xi d \eta
$$


Now, after evaluating the stiffness and mass matrices for all elements, the governing equations for free vibration analysis can be stated in the form of generalized eigenvalue problem.

$$
\left[K_{T}\right]\{\chi\}-\omega^{2}\left[M_{T}\right]\{\chi\}=0
$$

\section{Numerical results and discussions}

\subsection{Free vibration analysis of Square sandwich plate having three-ply laminated stiff sheets}

In this problem, a seven-layer simply supported square sandwich plate is studied. Two sandwich plates with various lay-ups on face sheets [0/90/0/core/0/90/0] and [45/-45/45/core/45/45/-45] are considered. The core is made of HEREX-C70.130 PVC foam and the face sheets are made of glass polyester resin. The mechanical properties of the sandwich plate are presented in Table 1 . The geometrical properties of the plate are $(a / h=10, a / b=1, h c / h=0.88)$ where $\mathrm{h}$ is the total thickness of the plate.

Table 1. Material properties for laminated sandwich plate.

\begin{tabular}{|c|c|c|c|c|c|c|c|}
\hline \multirow{2}{*}{ Material } & \multicolumn{7}{|c|}{ Elastic properties (GPa) } \\
\cline { 2 - 8 } & $E_{11}$ & $E_{22}$ & $G_{12}$ & $G_{13}$ & $G_{23}$ & $v_{12}$ & $\rho\left(\mathrm{Kg} / \mathrm{m}^{3}\right)$ \\
\hline \hline Faces & 24.51 & 7.77 & 3.34 & 3.34 & 1.34 & 0.078 & 1800 \\
\hline Core & 0.1036 & 0.1036 & 0.05 & 0.05 & 0.05 & 0.32 & 130 \\
\hline
\end{tabular}

The convergence of the non-dimensional results of natural frequencies, for the first four modes, is shown in Table 2 with different mesh sizes. The comparison was made with the analytical solutions based on LW approach (Jam et al., 2010; Rahmani et al., 2010), the 3D-finite element models also based on LW approach (FEM-3D-LW) given by Burlayenko et al. (2015). It is clear, from the table 2, that the results of developed element are in excellent agreement with numerical results found in the literature. These results show clearly the performances and convergence of the proposed layerwise formulation.

The non-dimensional results of frequencies are expressed as: $\bar{\omega}=\omega \frac{a^{2}}{h} \sqrt{\rho_{c} / E_{c}}$.

Table 2. Non-dimensional natural frequencies for a square multi-layered sandwich plate with various lay-ups on face sheets.

\begin{tabular}{|c|c|c|c|c|c|c|}
\hline \multirow{2}{*}{ References } & \multirow{2}{*}{ FE Models } & \multirow{2}{*}{$\begin{array}{l}\text { Various lay-ups } \\
\text { on face sheets }\end{array}$} & \multicolumn{4}{|c|}{ Frequencies (Hz) } \\
\hline & & & Mode 1 & Mode 2 & Mode 3 & Mode 4 \\
\hline Present element $(6 \times 6)$ & QSFT52 & \multirow{9}{*}{ Cas 1} & 14.736 & 28.207 & 28.802 & 37.584 \\
\hline Present element $(8 \times 8)$ & QSFT52 & & 14.583 & 27.499 & 28.115 & 36.627 \\
\hline Present element $(10 \times 10)$ & QSFT52 & & 14.513 & 27.173 & 27.796 & 36.167 \\
\hline Present element $(12 \times 12)$ & QSFT52 & & 14.477 & 26.999 & 27.626 & 35.954 \\
\hline Present element $(14 \times 14)$ & QSFT52 & & 14.452 & 26.893 & 27.524 & 35.777 \\
\hline Present element $(16 \times 16)$ & QSFT52 & & 14.440 & 26.826 & 27.456 & 35.706 \\
\hline Burlayenko et al. (2015) & FEM-3D-LW & & 14.620 & 26.800 & 27.400 & 35.550 \\
\hline Rahmani et al. (2010 & Analytical-LW & & 14.270 & 26.310 & 27.040 & 34.950 \\
\hline Jam et al. (2010) & Analytical-LW & & 15.040 & 26.733 & 27.329 & 35.316 \\
\hline Present element $(6 \times 6)$ & QSFT52 & \multirow{8}{*}{ Cas 2} & 15.674 & 28.756 & 28.756 & 38.363 \\
\hline Present element $(8 \times 8)$ & QSFT52 & & 15.536 & 28.069 & 28.069 & 37.478 \\
\hline Present element $(10 \times 10)$ & QSFT52 & & 15.473 & 27.754 & 27.754 & 37.053 \\
\hline Present element $(12 \times 12)$ & QSFT52 & & 15.437 & 27.587 & 27.587 & 36.805 \\
\hline Present element $(14 \times 14)$ & QSFT52 & & 15.419 & 27.485 & 27.485 & 36.698 \\
\hline Present element $(16 \times 16)$ & QSFT52 & & 15.405 & 27.417 & 27.417 & 36.592 \\
\hline Burlayenko et al. (2015) & FEM-3D-LW & & 15.420 & 27.170 & 27.460 & 36.240 \\
\hline Jam et al. (2010) & Analytical-LW & & 15.786 & 27.316 & 27.316 & 36.216 \\
\hline
\end{tabular}


Moreover, the same sandwich plate was analyzed for different thickness ratios $(a / h)$ and aspect ratios $(a / b)$ keeping the same ratios $h_{c} / h_{f}=0.88$. From Figure 3 , it is found that the nondimensional natural frequencies increase with increasing in thickness ratios, whatever the aspect ratios. This can be explained by the fact that the FRP sandwich plates are not assumed to be infinitely stiff through the thickness.

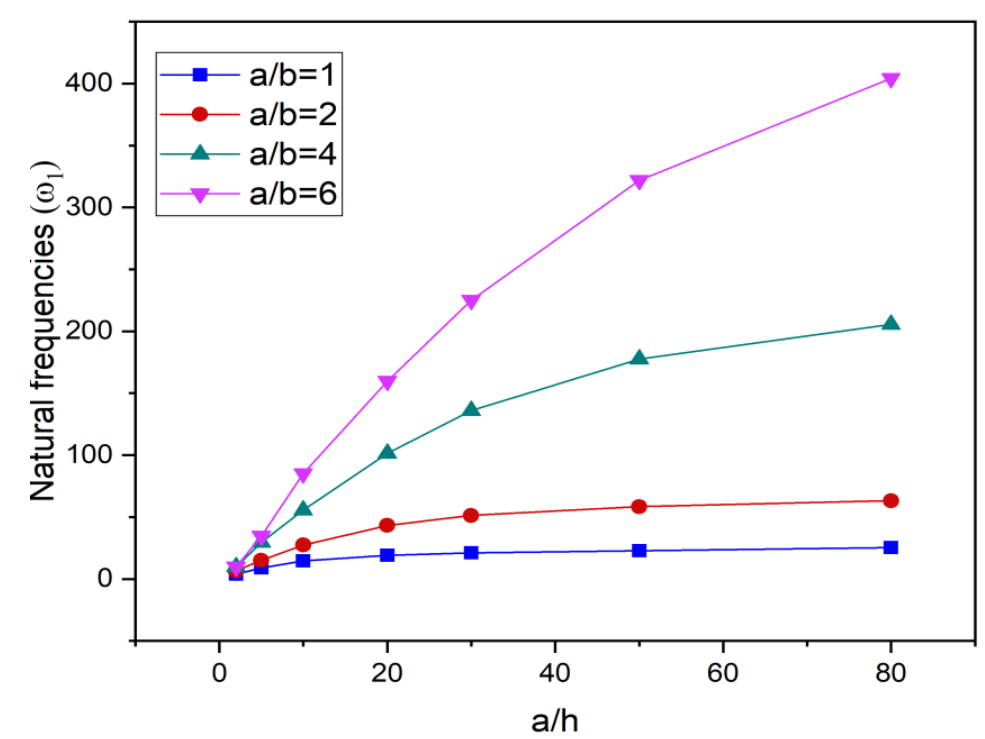

Fig. 3. Effect of a/h ratio on the fundamental frequencies of a simply supported square laminated sandwich plate $(0 / 90 / 0 / C / 0 / 90 / 0)$.

\subsection{Free vibration analysis of sandwich plate having un-symmetric laminated face sheets}

In this problem, a simply supported square sandwich plate with un-symmetric laminated face sheets $(0 / 90 / \mathrm{C} / 0 / 90)$ is considered to assess the performance of our model to the thin as well as thick plate. The mechanical properties of the sandwich plate are presented in table 3.The thickness ratio $(a / h)$ is considered to vary from 2 to 100 , where the ratio of thickness of core to thickness of face sheet $h_{c} / h_{f}$ is considered as 10 .

The first six mode shapes obtained for simply supported square laminated sandwich plate with $\mathrm{a} / \mathrm{h}=10$ are shown in Figures 4.

The non-dimensional results of frequencies are expressed as: $\bar{\omega}=\omega b^{2} / h \sqrt{\rho_{c} / E_{22 f}}$

The comparison of natural frequencies, considering mesh size $(12 \times 12)$, are shown in figure 5 , with those obtained by the 3D-elasticity solution (Rao et al., 2004), the analytical results based on HSDT (Kant and Swaminathan, 2001), and those obtained with the FEM-Q8 solution based on global-local higher order shear deformation theory (GLHSDT) (Zhen et al., 2010).

It can be seen, from the figures 4 and 5, that the present FE model gives more accurate results than the other models which confirm the good performance and robustness of the proposed formulation.

Table 3. Material properties for laminated sandwich plate.

\begin{tabular}{|c|c|c|c|c|c|c|c|}
\hline \multirow{2}{*}{ Material } & \multicolumn{7}{|c|}{ Elastic properties (GPa) } \\
\cline { 2 - 8 } & $E_{11}$ & $E_{22}$ & $G_{12}$ & $G_{13}$ & $G_{23}$ & $v_{12}$ & $\rho\left(\mathrm{Kg} / \mathrm{m}^{3}\right)$ \\
\hline \hline Faces & 131 & 10.34 & 6.9 & 6.9 & 6.9 & 0.22 & 1627 \\
\hline Core & 0.0069 & 0.0069 & 0.0034 & 0.0034 & 0.0034 & $10^{-5}$ & 97 \\
\hline
\end{tabular}




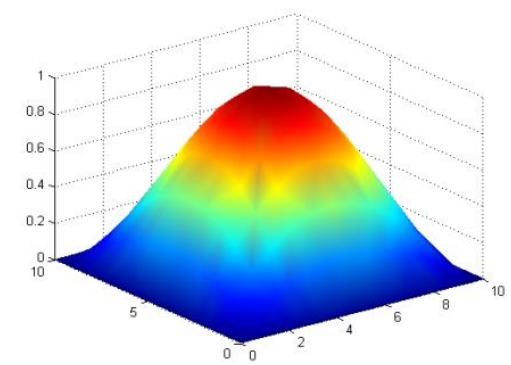

Mode 1

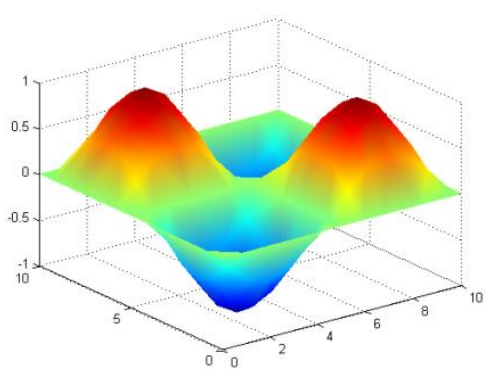

Mode 4

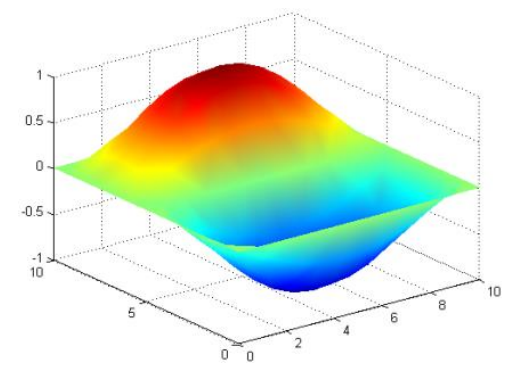

Mode 2

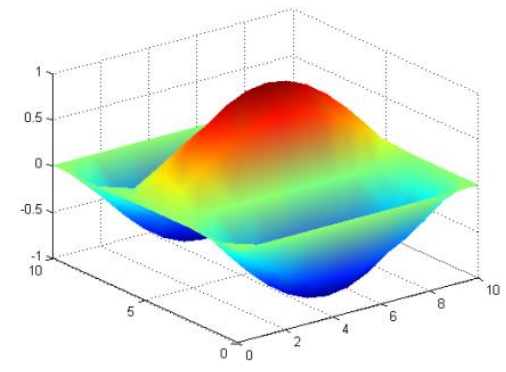

Mode 5

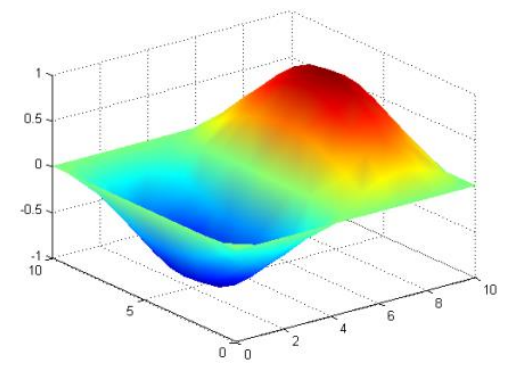

Mode 3

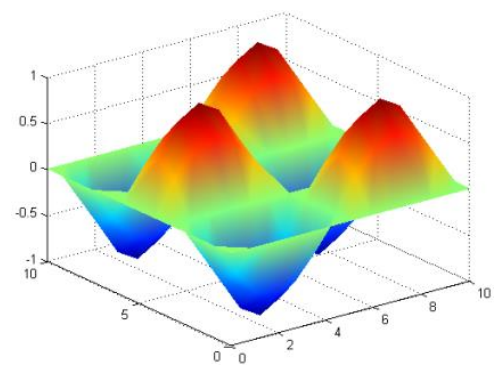

Mode 6

Fig. 4. First six mode shapes of simply supported square laminated sandwich plate $(0 / 90 / C / 0 / 90)$ with $\mathbf{a} / \mathbf{h}=10$.

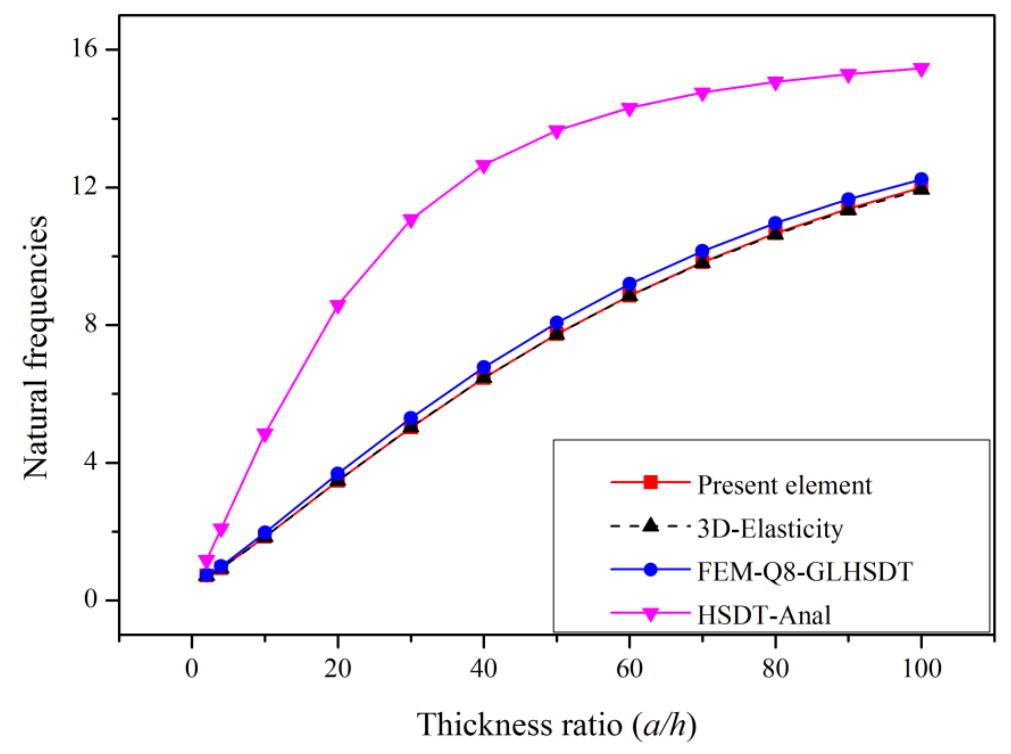

Fig. 5. Effect of the thickness ratios $(\mathrm{a} / \mathrm{h})$ on the non-dimensional fundamental frequencies of a simply supported sandwich plate having un-symmetric laminated face sheets.

\section{Conclusion}

In this paper, a new layerwise finite element model was proposed for the natural frequency analysis of multilayer sandwich plates. The developed model is based on a proper combination of higher-order and first-order, shear deformation theories. These combined theories satisfy interlaminar displacement continuity. The results obtained by our model were compared with those obtained by the analytical results and other finite element models found in literature. The comparison showed that the element has an excellent accuracy and a broad range of applicability. 
The effects of degree of length-to-thickness ratio $(\mathrm{a} / \mathrm{h})$ plate and aspect ratio $(\mathrm{a} / \mathrm{b})$ upon the fundamental frequencies are discussed and the results reaffirm that these effects plays an important role in the free vibration frequencies of laminated sandwich plates.

\section{References}

Araújo, A. L., Mota Soares, C. M., \& Mota Soares, C. A. (2010). Finite element model for hybrid activepassive damping analysis of anisotropic laminated sandwich structures. Journal of Sandwich Structures \& Materials, 12(4), 397-419.

Belarbi, M. O., \& Tati, A. (2015). A new C0 finite element model for the analysis of sandwich plates using combined theories. International Journal of Structural Engineering, 6(3), 212-239.

Belarbi, M. 0., \& Tati, A. (2016). Bending Analysis of Composite Sandwich Plates with Laminated Face Sheets: New Finite Element Formulation. Journal of Solid Mechanics, 8(2), 280-299.

Belarbia, M. O., Tatib, A., Ounisc, H., \& Benchabane, A. (2016). Development of a 2D isoparametric finite element model based on the layerwise approach for the bending analysis of sandwich plates. Structural Engineering and Mechanics, 57(3), 473-506.

Burlayenko, V. N., Altenbach, H., \& Sadowski, T. (2015). An evaluation of displacement-based finite element models used for free vibration analysis of homogeneous and composite plates. Journal of Sound and Vibration, 358, 152-175.

Desai, Y. M., Ramtekkar, G. S., \& Shah, A. H. (2003). Dynamic analysis of laminated composite plates using a layer-wise mixed finite element model. Composite structures, 59(2), 237-249.

Elmalich, D., \& Rabinovitch, O. (2012). A high-order finite element for dynamic analysis of soft-core sandwich plates. Journal of Sandwich Structures \& Materials, 14(5), 525-555.

Jam, J. E., Eftari, B., \& Taghavian, S. H. (2010). A new improved high-order theory for analysis of free vibration of sandwich panels. Polymer Composites, 31(12), 2042-2048.

Kant, T., \& Swaminathan, K. (2001). Analytical solutions for free vibration of laminated composite and sandwich plates based on a higher-order refined theory. Composite structures, 53(1), 73-85.

Nabarrete, A., M. De Almeida, S. F., \& Hansen, J. S. (2003). Sandwich-plate vibration analysis: three-layer quasi-three-dimensional finite element model. AIAA journal, 41(8), 1547-1555.

Pandey, S., \& Pradyumna, S. (2015). A new C0 higher-order layerwise finite element formulation for the analysis of laminated and sandwich plates. Composite Structures, 131, 1-16.

Rahmani, O., Khalili, S. M. R., \& Malekzadeh, K. (2010). Free vibration response of composite sandwich cylindrical shell with flexible core. Composite Structures, 92(5), 1269-1281.

Rao, M. K., Scherbatiuk, K., Desai, Y. M., \& Shah, A. H. (2004). Natural vibrations of laminated and sandwich plates. Journal of Engineering Mechanics, 130(11), 1268-1278.

Zhen, W., Wanji, C., \& Xiaohui, R. (2010). An accurate higher-order theory and C0 finite element for free vibration analysis of laminated composite and sandwich plates. Composite Structures, 92(6), 1299-1307. 